\title{
A Study of Quantum Confinement Effects in Ultrathin NiO Films Performed by Experiment and Theory
}

\author{
Christos S. Garoufalis ${ }^{1}$, Alexandros Barnasas ${ }^{1}$, Alkeos Stamatelatos ${ }^{1}$ (i), Vagelis Karoutsos ${ }^{1}$, \\ Spyridon Grammatikopoulos ${ }^{2}$ (D), Panagiotis Poulopoulos ${ }^{1}$ and Sotirios Baskoutas ${ }^{1, *}$ (D) \\ 1 Department of Materials Science, University of Patras, 26504 Patras, Greece; garoufal@upatras.gr (C.S.G.); \\ mparnalex@gmail.com (A.B.); alkstamatelatos@gmail.com (A.S.); vkar@upatras.gr (V.K.); \\ poulop@upatras.gr (P.P.) \\ 2 Department of Mechanical Engineering, Technological Educational Institute (T.E.I.) of Western Greece, \\ M. Alexandrou 1, 26504 Patras, Greece; spiridongramma@upatras.gr \\ * Correspondence: bask@upatras.gr; Tel./Fax: +30-2610-96-9349
}

Received: 5 March 2018; Accepted: 1 June 2018; Published: 4 June 2018

\begin{abstract}
Ultrathin $\mathrm{NiO}$ films in the thickness range between 1 and $27 \mathrm{~nm}$ have been deposited on high-quality quartz substrates by direct magnetron sputtering under a rough vacuum with a base pressure of $2 \times 10^{-2}$ mbar. The sputtering target was metallic $\mathrm{Ni}$; however, due to the rough vacuum a precursor material was grown in which most of Ni was already oxidized. Subsequent short annealing at temperatures of about $600{ }^{\circ} \mathrm{C}$ in a furnace in air resulted in $\mathrm{NiO}$ with high crystallinity quality, as atomic force microscopy revealed. The images of surface morphology showed that the $\mathrm{NiO}$ films were continuous and follow a normal grain growth mode. UV-Vis light absorption spectroscopy experiments have revealed a blue shift of the direct band gap of $\mathrm{NiO}$. The band gap was determined either by Tauc plots (onset) or by the derivative method (highest rate of absorbance increase just after the onset). The experimental results are interpreted as evidences of quantum confinement effects. Theoretical calculations based on Hartree Fock approximation as applied for an electron-hole system, in the framework of effective mass approximation were carried out. The agreement between theory and experiment supports the quantum confinement interpretation.
\end{abstract}

Keywords: thin films; semiconductor oxides; grain growth; optical properties; quantum confinement; potential morphing method

\section{Introduction}

$\mathrm{NiO}$ is a wide direct band gap semiconductor with an energy band gap $E_{g}$ close to $4 \mathrm{eV}$ [1]. $\mathrm{NiO}$ is placed in the center of scientific attention for the realization of various applications, such as gas sensing [2,3], nonvolatile storage media [4], battery cathodes [5], and transparent UV detectors in the field of optoelectronics [6]. Moreover, it can be used in photovoltaics as a transparent oxide semiconductor $[7,8]$ and in the formation of modern pn junctions, such as $\mathrm{NiO} / \mathrm{ZnO}$ ones $[9,10]$. Among the most important properties of a nanoscaled semiconductor for optoelectronics is the controllable band gap tuning [11,12]. This tuning is achievable through the strong band gap dependence on the particle size or film thickness of the semiconductors and, therefore, it can be tailored by reducing the semiconductor dimensionality.

There are many studies dealing with the $E_{g}$ determination of $\mathrm{NiO}$ thin films and nanoparticles. In most of them, $E_{g}$ is found to be close to the bulk value, while there are also reports which indicate a "red" shift (towards lower energies) of $E_{g}$ (see e.g., [13-16]). However, due to the very small Bohr radius of $\mathrm{NiO}$, it is difficult to find studies where a "blue" shift systematically develops with reduced dimensionality and investigated by experiment and theory. Some preliminary results were published 
earlier [17]. In the present contribution, we present a complete experimental study which is also supported and complemented by theoretical calculation based on the potential morphing method (PMM). We grow ultrathin $\mathrm{NiO}$ thin films with an alternative method which starts from the growth of a precursor $\mathrm{NiO}$ material in a rough vacuum followed by a short annealing time at $600{ }^{\circ} \mathrm{C}$ [18]. At such elevated temperatures $\mathrm{NiO}$ film crystallinity improves [19]. The growth habits of our $\mathrm{NiO}$ films with thicknesses ranging from 1 to $27 \mathrm{~nm}$ are briefly presented and discussed. Subsequently, our focus is turned on their optical absorption properties, which are found to exhibit a smooth increase of the $E_{g}$ position as the film thickness decreases. The maximum value of the blue shift observed in our experiments is around $0.4 \mathrm{eV}$ and it corresponds to our thinnest $(1 \mathrm{~nm}) \mathrm{NiO}$ sample. The observed trends remain the same regardless of the adopted method of study (i.e., Tauc plots [20] or derivative method [12]). These two different approaches are compared and discussed in a comprehensive way.

The results are compared to theoretical calculations based on a two-particle (electron and hole) Hartree Fock (HF) approximation [21-23], which is solved in the framework of effective mass approximation (EMA). This choice of method is dictated not only by the fact that the size of the systems under consideration is by far too large for more robust and advanced approximation (e.g., DFT), but also because a detailed microscopic treatment exhibits difficulties (due to passivation issues) in capturing the quantum confinement effect. For example, it is well known that the (111) surface of $\mathrm{NiO}$ can adopt several types of reconstruction such as the so-called octopolar, $2 \times 2-\alpha$, and Rt3, which, in a microscopic theoretical treatment, must be explicitly considered. On top of this, in such a treatment, the passivation of the surface has to be done atom by atom and it might have a significant effect on the electronic properties (and the calculated gap) of the material. This becomes evident in the thorough and extensive DFT + U investigation of (111) NiO surface by Li et al. [24], which revealed that the calculations on three slabs with different thicknesses $(1.43,1.92$, and $2.41 \mathrm{~nm})$ produced the same gap $\sim 1.19 \mathrm{eV}$ which, by the way, is also smaller than the calculated bulk gap $(2.43 \mathrm{eV})$. As a result, we resort to the traditional and well behaved combination of HF and EMA, which has a proven record of successful treatments of such problems. The solution of the relevant equations is achieved numerically with the help of the potential morphing method (PMM) [25]. It should be noted that $\mathrm{NiO}$ is a natural p-type semiconductor due to deviations from stoichiometry [26]. Taking this into consideration, as well as the fact that the effective mass of doped semiconductors increases [27], we perform suitable calculations in order to study the influence of this effect on the "blue' shift of $E_{g}$. Our theoretical results for the dependence of the direct band gap of $\mathrm{NiO}$ on the film thickness are in good agreement with the experimental values. This agreement verifies that the experimentally-observed shifts can be safely attributed to quantum confinement effects.

\section{Materials and Methods}

\subsection{Experimental Details}

Ultrathin $\mathrm{NiO}$ films with thicknesses in the range of 1-27 nm were deposited on quartz glass by direct current (DC) magnetron sputtering. The Ni target was $99.95 \%$ pure and was purchased by Alfa Aesar ((Karlsruhe, Germany). The deposition temperature was the room temperature. We used a simple table sputter coater by Balzers (Balzers Union model SCD040, Liectenstein). Therefore, the base pressure of the vacuum chamber was $2 \times 10^{-2} \mathrm{mbar}$. The total pressure (including Ar partial pressure) during deposition was about $5 \times 10^{-2}$ mbar.

Film thickness evaluation was performed with the help of AFM, Multimode, (Bruker, Santa Barbara, CA, USA) images of the profile of a narrow scratch done intentionally on the film surface [28]. The AFM was a multimode microscope with a Nanoscope IIIla controller and a $120 \mu \mathrm{m} \times 120 \mu \mathrm{m}$ magnet-free scanner (model AS-130VMF) developed by Bruker (Santa Barbara, CA, USA). The microscope was operated in the non-contact (tapping) mode [29].

As we have recently shown for $\mathrm{Au}-\mathrm{Ni}-\mathrm{O}$ films [18], under such growth conditions in a rough vacuum, a precursor thin film material is formed. This may contain a major part of $\mathrm{NiO}$ and to 
a less extent metallic Ni. In this work, selected samples were measured via energy dispersive X-ray spectroscopy (EDS) with our scanning electron microscope (SEM) a Zeiss EVO MA 10 model (ZEISS, Oberkochen, Germany). It was found that the ratio of $\mathrm{Ni}$ and $\mathrm{O}$ atoms within the experimental error was almost 1, as it should be in NiO. In order to obtain high-quality nanocrystalline samples [19], and strain annihilation, the films were subsequently annealed for $20 \mathrm{~min}$ at $600{ }^{\circ} \mathrm{C}$ in air. The films' microstructure was then monitored by AFM experiments.

Finally, the ultraviolet UV-Vis spectra were recorded at room temperature in the transmission geometry with the help of a Perkin Elmer $\Lambda$-35 UV-Vis spectrometer (PerkinElmer, Akron Ohio, USA) at the wavelength range $200-1100 \mathrm{~nm}$.

\subsection{Theory}

In the effective mass approximation the Hamiltonian for the electron hole system can be written as [21-23]:

$$
H=-\frac{\hbar^{2}}{2 m_{e}^{*}} \nabla_{e}^{2}-\frac{\hbar^{2}}{2 m_{h}^{*}} \nabla_{h}^{2}+V_{0}^{e}\left(r_{e}\right)+V_{0}^{h}\left(r_{h}\right)-\frac{e^{2}}{\varepsilon} \frac{1}{r_{e h}}
$$

where $m_{e}^{*}\left(m_{h}^{*}\right)$ is the effective electron (hole) band mass, $\varepsilon$ is the size dependent effective dielectric constant [30], $r_{e h}$ is the electron-hole distance and $V_{0}^{e(h)}$ is the confinement potential of electron (hole). The Hartree-Fock formulation of two particles (electron and one hole) results in the following coupled equations:

$$
\left[\frac{p_{i}^{2}}{2 m_{i}^{*}}+U_{i}\left(r_{i}\right)\right] \Phi_{i}\left(r_{i}\right)=\widetilde{E}_{i} \Phi_{i}\left(r_{i}\right) \quad i=e, h
$$

The self-consistent effective field $U_{i}\left(r_{i}\right)$ that acts on the particles includes the interaction with the confining potential as well as the Coulomb and exchange interaction between the two particles [21-23]. Once the numerical solution has been achieved [21-23], the total energy of the exciton, is calculated as $E(X)=\widetilde{E}_{e}+\widetilde{E}_{h}$, while the corresponding effective band gap is obtained by adding the exciton energy to the band gap of the bulk material:

$$
E_{g}^{e f f}(X)=E_{g}+E(X)
$$

A more detailed description of the adopted methodology (including technical details of the calculations) can be found elsewhere [21-23]. In the specific implementation, the shape of the confining potential, was assumed to be $V_{0}$ outside the film and zero inside. The value of $V_{0}$ is empirically determined as described in previous studies [22]:

$$
V_{0}^{e(h)}= \begin{cases}0 & |z|<L / 2 \\ V_{0} & |z| \geq L / 2\end{cases}
$$

\section{Results}

\subsection{Grain Growth}

Atomic force microscopy (AFM, Multimode, Bruker, Santa Barbara, CA, USA) experiments have been performed for all films with thickness $t$ ranging between 4 and $27 \mathrm{~nm}$. The resolution of the AFM was not adequate enough to probe thinner films. In Figure 1a one may see, as a typical example, the AFM image from the surface of a $13.5 \mathrm{~nm}$ thick $\mathrm{NiO}$ film. It is noteworthy that our AFM image unambiguously shows a facetted morphology of $\mathrm{NiO}$ and nanocrystals with good symmetric shape. This is a strong evidence of sample crystallinity. A similar AFM image from a $4 \mathrm{~nm}$ thick $\mathrm{NiO}$ film is also presented in Figure 1b. Both films are smooth and continuous. The surface roughness is relatively small and the nanocrystallites (grains) seem to be quite homogeneous in terms of size. In Figure 1c,d we plot the grain diameter $D$ size distribution of the two films of Figure $1 \mathrm{a}, \mathrm{b}$, respectively. One may 
observe that both distributions are well-described by the log-norm function [31]. The average values of $D$ are $16.8 \mathrm{~nm}$ and $7.7 \mathrm{~nm}$ for the 13.5 and $4 \mathrm{~nm}$ thick films. The error in the determination of the grain size increases for thinner films as $D$ approaches the tip radius of $7 \mathrm{~nm}$. The full-width at half-maximum (FWHM) is $12.1 \mathrm{~nm}$ for the first film and $4.7 \mathrm{~nm}$ for the second. Therefore, both distributions are relatively narrow.
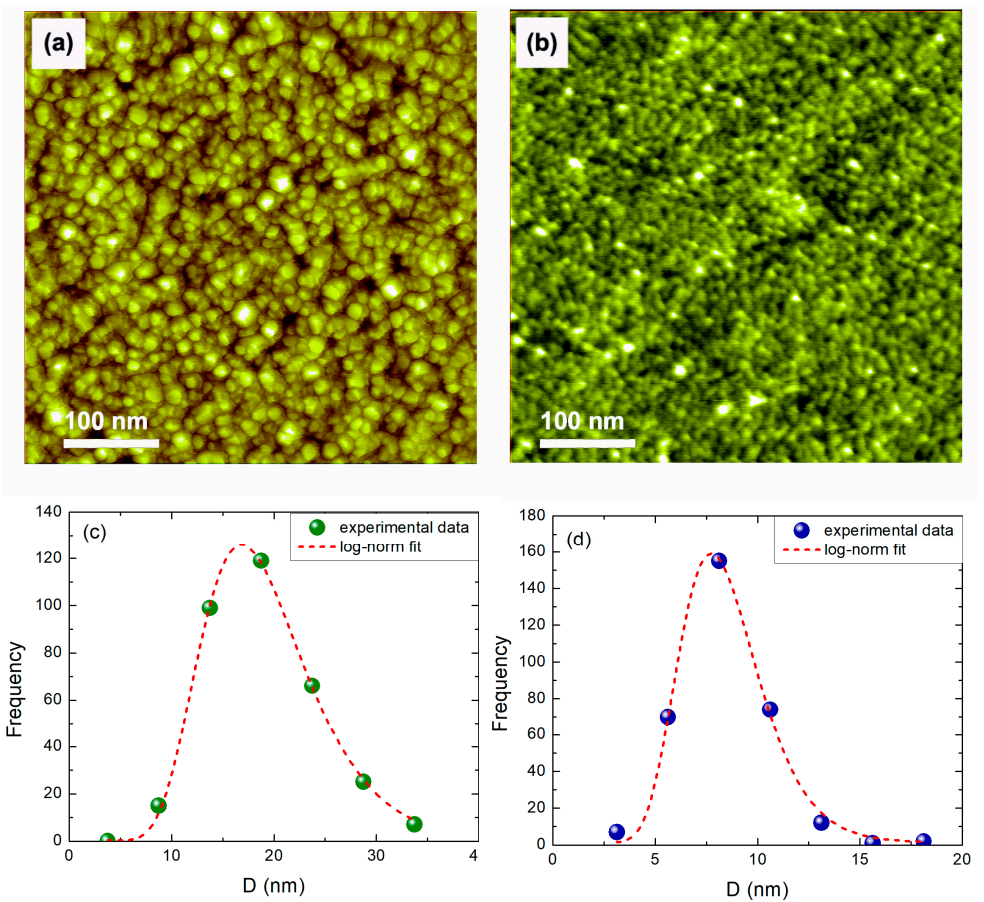

Figure 1. AFM images of (a) $13.5 \mathrm{~nm}$ and (b) $4 \mathrm{~nm}$ thick NiO films. The image size is $500 \times 500 \mathrm{~nm}^{2}$. Grain diameter $D$ size distributions for the (c) $13.5 \mathrm{~nm}$ and (d) $4 \mathrm{~nm}$ thick $\mathrm{NiO}$ films.

In Figure 2a we attempted to plot the log-log plot for the grain diameter as a function of film thickness. This type of plots may provide some indirect information on the grain growth mode of thin films [32-35]. The slope of the line after linear regression is $0.49 \pm 0.09$. This value is very close to the theoretical and computational values for normal grain growth mode [36,37]. One has to recall that our films' images were recorded after short annealing at a relatively high temperature. This temperature of $873 \mathrm{~K}$, however, is much smaller than the melting point of $\mathrm{NiO}$ which is about $2228 \mathrm{~K}$. Therefore, we are far below the recrystallization temperature. $\mathrm{NiO}$ grains in our films may become larger after short annealing than in the as-deposited state, but they do not grow the one in the expense of the other; the latter is typical at the recrystallization temperature and above. Thus, the normal grain growth feature of homogeneous monomodal grain-size distribution is maintained. This is confirmed both, by the AFM images of Figures 1 and 2a.

Average root-mean-square roughness values, $R_{\mathrm{rms}}$, as a function of film thickness for the five $\mathrm{NiO}$ samples studied by AFM is plotted in Figure $2 \mathrm{~b}$. According to the log-log plot of this figure the roughness increases with thickness following a power law with the form $R_{\mathrm{rms}} \sim t^{\mathrm{b}}$, where $b$ is the slope of the best fitted line which is found to be $b=0.54 \pm 0.12$. The aforementioned power law has been predicted by many theoretical models for polycrystalline thin films $[38,39]$, where the exponent $b$ is in the range of $0.3-0.9$ (see, for example, [34]). Surface roughness measurements made on the same system, i.e., $\mathrm{NiO}$ films, grown on $\mathrm{Si}$ wafers at $200{ }^{\circ} \mathrm{C}$ by atomic layer deposition, with a similar thickness as our films (6-29 nm), resulted in a $b=0.94$ [40]. We notice that there is a significant deviation with the exponent value obtained by our measurements. However, we have to bear in mind that the roughness data are nothing more but statistical values about the surface height information and these 
value depend on many factors, such as the deposition technique, film substrate, substrate temperature, as well as which surface area is studied [34].
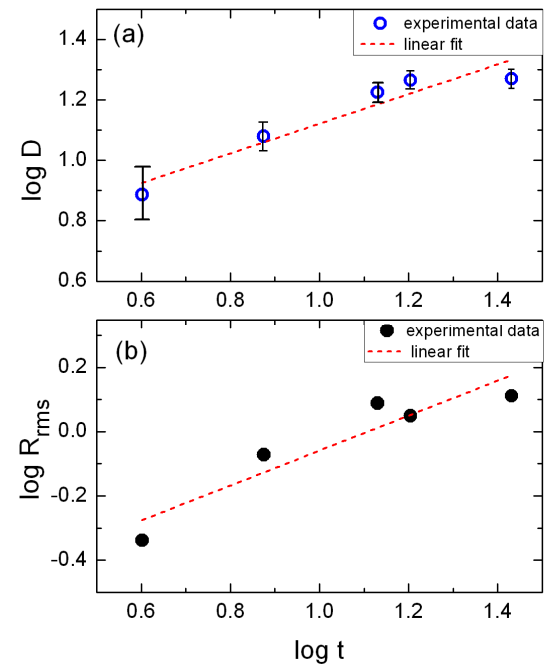

Figure 2. (a) Grain diameter D as a function of film thickness $t$ in a log-log plot. The slope of the linear fit has a value consistent with normal grain growth mode. The error bar increases with decreasing film thickness due to the finite tip radius of $7 \mathrm{~nm}$. (b) root-mean-square roughness values as a function of film thickness for the same five $\mathrm{NiO}$ samples the thickness of which is in the range of 4-27 nm.

\subsection{Optical Properties}

In Figure $3 a$ we plot the optical density (or absorbance $A=-\log T, T$ is the transmittance [20]) spectra, for two $\mathrm{NiO}$ films. In Figure $3 b$ the first derivative of $A$ is shown. The first derivative maximum marks the point where one encounters the highest rate of the absorbance increase just after the onset of $E_{g}$. The arrows indicate a clear "blue" shift of the spectra and of the derivative maxima as the film thickness decreases. Although there is a debate on the use of either the derivative, or the more commonly used Tauc plots, for the study of the blue shift of the energy gap and the features of the absorbance spectra, the current $\mathrm{NiO}$ samples give us an excellent opportunity to go into some more details since the spectra are almost free of noise.

Therefore, for the two aforementioned films ( $t=13.5 \mathrm{~nm}$ and $t=2 \mathrm{~nm}$ ) we present in Figure 4 , two commonly adopted approaches of a Tauc plot for a semiconductor with direct $E_{g}$ and dipole-dipole allowed transitions. In particular, Figure $4 \mathrm{a}$ shows the magnitude of $(\alpha E)^{2}$ as a function of photon energy E, e.g., [13-16], while Figure 4 b depicts the variation of $\alpha^{2}$ as a function of $E$ [20] ( $\alpha$ is the absorption coefficient). One may observe that there is a large almost linear part of the curves near the onset of $E_{g}$. The intercept with the energy axis is $E_{g}$. In most cases in literature, one deals with relatively thick films. Rayleigh scattering at the grain boundaries, defects or impurities add a parabolic slope to the whole spectrum of the absorbance [20]. This effect limits the linearity of the Tauc plots. Therefore, they exhibit a degree of uncertainty depending on how straight a Tauc curve is. On the contrary, in most cases, the derivative method is found to be more accurate since the maximum of $d A / d E$ can be safely determined. In our case the quality of the data, which reflects the quality of the films, makes the linear part of Tauc plots significant, see Figure 4, and increases the accuracy of the determined onset of $E_{g}$.

The results of the derivative method and the two Tauc plots are collected and presented in a comparative manner in Figure 5. Moreover, with the lines, we present the sum of the onset of $E_{g}$ determined by the Tauc plots and a vertical shift constant. We see that the two lines coincide, within the experimental accuracy, with the derivative data. Therefore, it is safe to conclude that all these values of the optical-properties (i.e., $E_{g}$ onset and maximum absorbance increase rate) exhibit the same behavior (i.e., they increase as the film thickness decreases). Indeed, this becomes even more clear if one goes to 
the thinner films where quantum confinement effects are enhanced since the film thickness becomes comparable to the Bohr radius of the excitons.
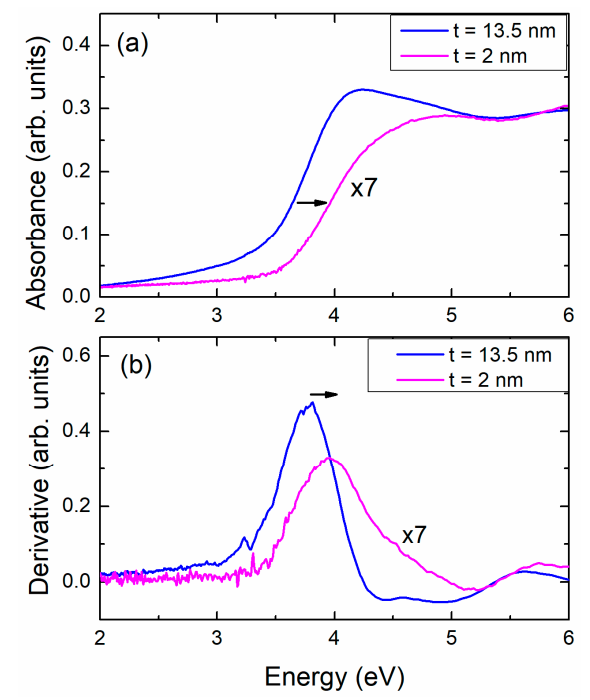

Figure 3. (a) Absorbance spectra for two NiO films. The film thickness is indicated. The absorbance of the thinner film has been multiplied by 7 for better clarity. One may see a "blue" shift of the energy band gap as determined by the first derivative of the spectra, see (b).
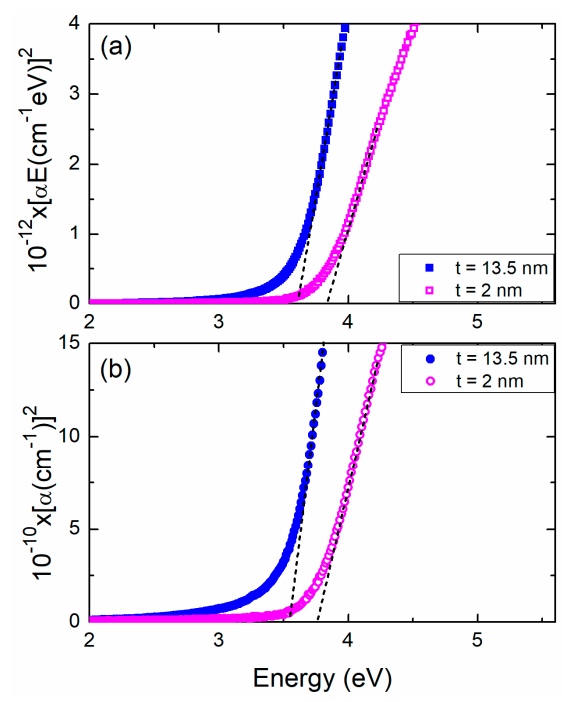

Figure 4. Tauc plots for two NiO films. (a) $(\alpha E)^{2}$ as a function of photon energy $E$ and (b) $(\alpha)^{2}$ as a function of E. One can clearly observe a "blue" shift of $E_{g}$ determined with high precision by the extrapolation of a rather large linear part of the plots. The film thickness is indicated. 


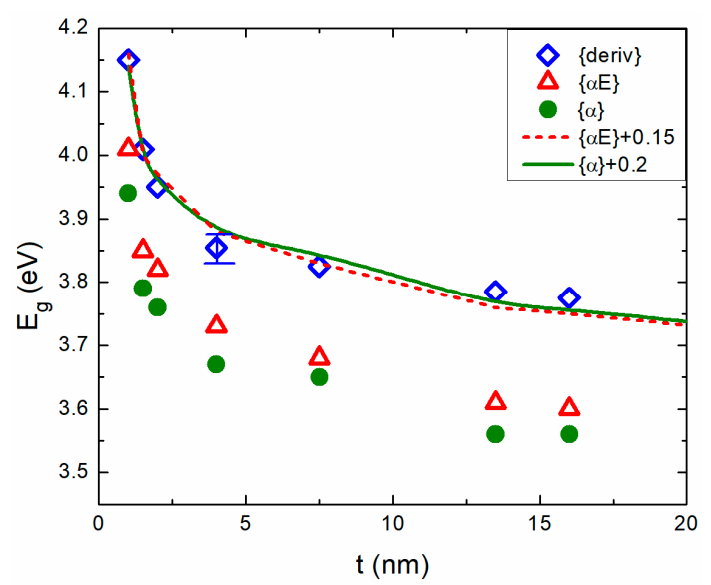

Figure 5. All features of absorbance spectra such as the onset of $E_{g}$ determined by the Tauc plots and the maximum of absorbance after the onset determined by the derivative maximum, coincide.

For completeness sake, in Figure 6 we present the absorption coefficient determination by the slope of a $(-\ln T)$ as a function of film thickness t diagram. We select a photon energy of $5 \mathrm{eV}$ (wavelength $\lambda$ of $250 \mathrm{~nm}$ ), because this energy position in all spectra, even the "blue" shifted ones, is after the edge jump. Our value of $\alpha$ at $250 \mathrm{~nm}$ is equal to $5 \times 10^{5} \mathrm{~cm}^{-1}$, which is in fair agreement with previous reports on high-quality films [1].

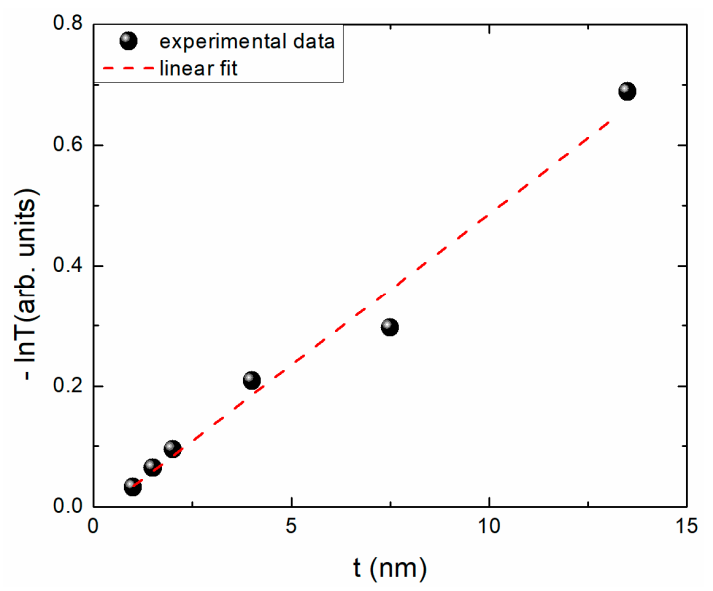

Figure 6. $(-\ln T)$ as a function of film thickness $t$. The slope of the linear fit is equal to the absorption coefficient. The transmittance $\mathrm{T}$ data have been recorded at $5 \mathrm{eV}(250 \mathrm{~nm})$.

\section{Discussion and Conclusions}

Any calculation based on the effective mass approximation is heavily dependent on the supplied values of electron and hole effective masses $\left(m_{e}^{*}, m_{h}{ }^{*}\right)$. However, for the case of $\mathrm{NiO}$, the values found in the literature appear to be extremely diverse. It is worth noting that in many cases, even the reported trends, are not consistent with each other [27,41-44]. To some extent this diversity might be related to changes in the conduction properties (i.e., p-type semiconductor, etc.) which are affected by variations in stoichiometry and, in turn, might also modify the value of the masses. As a result, we performed several benchmark calculations following the diverse literature suggestions, until we finally adopted the conclusion of Irwin et al. [27] who propose that the electron and hole exhibit similar masses which range from 0.5 to $1.0 m_{0}$ (i.e., $m_{e}{ }^{*} \approx m_{h}{ }^{*}=0.5-1.0 m_{0}$ ). Furthermore, in order to include the dependence of the dielectric constant on the film thickness we have chosen to incorporate into our 
model the size-dependent dielectric function of Hanken [45] with the following parameters: $\varepsilon_{\infty}=5.7$, $\varepsilon_{0}=12.4$ [46] and $\omega_{\mathrm{LO}}=64.47 \mathrm{meV}$ [47].

The results for $m_{e}^{*} \approx m_{h}^{*}=0.8 m_{0}$ are presented in Figure 7 along with experimental data. It is evident that the agreement between theory and experiment is quite good. It is worth noting that when the PMM values (produced by all combinations of masses) are fitted on $E_{g}=a+b / t^{\mathrm{c}}$ formula, the $c$ exponent ranges from 1.5 to 1.7 , while for the experimental data the $c$ parameter is closer to 1.0 (in simple effective mass theory, $c$ is expected to be 2.0). In conclusion, the apparent similarity of the two curves presented in Figure 7 is highly suggestive that the observed shifts are a manifestation of the quantum confinement effect.

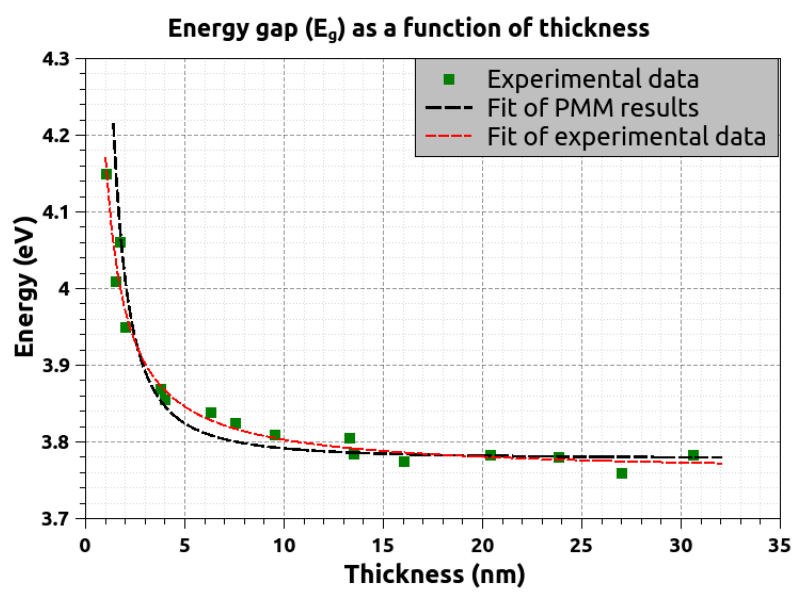

Figure 7. Direct band gap energy $E_{g}$ of ultrathin $\mathrm{NiO}$ films as a function of film thickness $t$ by experiment and theory. Some experimental data from Ref. [17] have been also included. The black dashed line is derived by fitting the calculated values of $E_{g}$ on $E_{g}=a+b / t^{c}(a, b$, and $c$ are fitting parameters and $t$ is the film thickness) while the red dashed line is a fit of the experimental data on the same function.

In this work we have studied microstructural features, i.e., grain size and surface roughness as a function of thickness up to $27 \mathrm{~nm}$ for ultrathin $\mathrm{NiO}$ films grown by direct current magnetron sputtering in rough vacuum and annealed shortly at $600{ }^{\circ} \mathrm{C}$. Moreover, we have recorded the absorbance spectra of these films. We determine the onset of the energy band gap $E_{g}$ by using Tauc plots and the maximum rate of the absorbance increase after $E_{g}$ by the derivative method. Both features show a smooth increase, a "blue" shift, with decreasing NiO film thickness down to $1 \mathrm{~nm}$. The experiment is well described by effective mass Hartree Fock calculations revealing that the blue shift is due to quantum confinement.

Author Contributions: C.S.G. performed the calculations and wrote parts of the article; A.B. performed the film growth and did the statistics on crystal growth; A.S. performed the film growth and optical characterization; V.K. performed the AFM characterization and wrote part of the article; S.G. supervised the film growth; P.P. supervised parts of the AFM and optical study and wrote parts of the article; and S.B. supervised the theoretical work and conducted discussions.

Funding: This research was partially funded by the Andreas Mentzelopoulos Scholarships for the University of Patras.

Conflicts of Interest: The authors declare no conflict of interest.

\section{References}

1. Powell, J.; Spicer, W.E. Optical Properties of NiO and CoO. Phys. Rev. B 1970, 2, 2182-2193. [CrossRef]

2. Hotovy, I.; Rehacek, V.; Siciliano, P.; Capone, S.; Spiess, L. Sensing characteristics of $\mathrm{NiO}$ thin films as $\mathrm{NO}_{2}$ gas sensor. Thin Solid Films 2002, 418, 9-15. [CrossRef] 
3. Wang, J.; Yang, P.; Wei, X.; Zhou, Z. Nano- and mesoscale modeling of cement matrix. Nanoscale Res. Lett. 2015, 10, 173. [CrossRef]

4. Wang, H.; Zou, C.; Zhou, L.; Tian, C.; Fu, D. Resistive switching characteristics of thin NiO film based flexible nonvolatile memory devices. Microelectron. Eng. 2012, 91, 144-146. [CrossRef]

5. Bogner, M.; Fuchs, A.; Scharnagl, K.; Winter, R.; Doll, T.; Eisele, I. Thin $(\mathrm{NiO})_{1-x}\left(\mathrm{Al}_{2} \mathrm{O}_{3}\right)_{x}, \mathrm{Al}$ doped and $\mathrm{Al}$ coated $\mathrm{NiO}$ layers for gas detection with HSGFET. Sens. Actuators B Chem. 1998, 47, 145-152. [CrossRef]

6. Ohta, H.; Hosono, H. Transparent oxide optoelectronics. Mater. Today 2004, 7, 42-51. [CrossRef]

7. Irwin, M.D.; Buchholz, D.B.; Hains, A.W.; Chang, R.P.H.; Marks, T.J. P-Type semiconducting nickel oxide as an efficiency-enhancing anode interfacial layer in polymer bulk-heterojunction solar cells. Proc. Natl. Acad. Sci. USA 2008, 105, 2783-2787. [CrossRef]

8. Park, J.H.; Seo, J.; Park, S.; Shin, S.S.; Kim, Y.C.; Jeon, N.J.; Shin, H.; Ahn, T.K.; Noh, J.H.; Yoon, S.C.; et al. Efficient $\mathrm{CH}_{3} \mathrm{NH}_{3} \mathrm{PbI}_{3}$ perovskite solar cells employing nanostructured p-type $\mathrm{NiO}$ electrode formed by a pulsed laser deposition. Adv. Mater. 2015, 27, 4013-4019. [CrossRef] [PubMed]

9. Chrissanthopoulos, A.; Baskoutas, S.; Bouropoulos, N.; Dracopoulos, V.; Poulopoulos, P.; Yannopoulos, S.N. Synthesis and characterization of $\mathrm{ZnO} / \mathrm{NiO}$ p-n heterojunctions: $\mathrm{ZnO}$ nanorods grown on $\mathrm{NiO}$ thin film by thermal evaporation. Photonics Nanostruct. 2011, 9, 132-139. [CrossRef]

10. Dewan, S.; Tomar, M.; Tandon, R.P.; Gupta, V. Zn doping induced conductivity transformation in NiO films for realization of p-n homo junction diode. J. Appl. Phys. 2017, 121, 215307-215316. [CrossRef]

11. Chen, L.; Shet, S.; Tang, H.; Ahn, K.; Wang, H.; Yan, Y.; Turner, J.; Al-Jassim, M. Amorphous copper tungsten oxide with tunable band gaps. J. Appl. Phys. 2010, 108, 043502. [CrossRef]

12. Baskoutas, S.; Poulopoulos, P.; Karoutsos, V.; Angelakeris, M.; Flevaris, N.K. Strong quantum confinement effects in thin zinc selenide films. Chem. Phys. Lett. 2006, 417, 461-464. [CrossRef]

13. Sawaby, A.; Selim, M.S.; Marzouk, S.Y.; Mostafa, M.A.; Hosny, A. Structure, optical and electrochromic properties of NiO thin films. Physica B 2010, 405, 3412-3420. [CrossRef]

14. Subramanian, B.; Mohamed Ibrahim, M.; Senthilkumar, V.; Murali, K.R.; Vidhya, V.S. Optoelectronic and electrochemical properties of nickel oxide $(\mathrm{NiO})$ films deposited by DC reactive magnetron sputtering. Physica B 2008, 403, 4104-4110. [CrossRef]

15. Ush, K.S.; Sivakumar, R.; Sanjeeviraja, C. Optical constants and dispersion energy parameters of NiO thin films prepared by radio frequency magnetron sputtering technique. J. Appl. Phys. 2013, 114, 123501-123511. [CrossRef]

16. Jiang, D.Y.; Qin, J.M.; Wang, X.; Gao, S.; Liang, Q.C.; Zhao, J.X. Optical properties of NiO thin films fabricated by electron beam evaporation. Vacuum 2012, 86, 1083-1086. [CrossRef]

17. Pappas, S.D.; Grammatikopoulos, S.; Poulopoulos, P.; Trachylis, D.; Velgakis, M.J.; Politis, C. Growth and Optical Properties of Thin NiO Films. J. Surf. Interfaces Mater. 2014, 2, 233-237. [CrossRef]

18. Grammatikopoulos, S.; Stamatelatos, A.; Delimitis, A.; Sousanis, A.; Chrisanthopoulou, A.; Trachylis, D.; Politis, C.; Poulopoulos, P. Growth of Au Nanoparticles in NiO via Short Annealing of Precursor Material Thin Film and Optimization of Plasmonics. Phys. Status Solidi A 2017, 214, 1700303-1700309. [CrossRef]

19. Fasaki, I.; Koutoulaki, A.; Kompitsas, M.; Charitidis, C. Structural, electrical and mechanical properties of $\mathrm{NiO}$ thin films grown by pulsed laser deposition. Appl. Surf. Sci. 2010, 257, 429-433. [CrossRef]

20. Fox, M. Optical Properties of Solids, 2nd ed.; Oxford University Press: New York, NY, USA, 2010; ISBN 9780199573370.

21. Rieth, M.; Schommers, W.; Baskoutas, S. Exact numerical solution of Schroedinger's equation for a particle in an interaction potential of general shape. Int. J. Mod. Phys. B 2002, 16, 4081-4092. [CrossRef]

22. Baskoutas, S.; Terzis, A.F.. Size-dependent band gap of colloidal quantum dots. J. Appl. Phys. 2006, 99, 013708-013712. [CrossRef]

23. Baskoutas, S.; Terzis, A.F.; Schommers, W. Size-dependent exciton energy of narrow band gap colloidal quantum dots in the finite depth square-well effective mass approximation. J. Comp. Theor. Nanosci. 2006, 3, 269-271. [CrossRef]

24. Li, L.; Kanai, Y. Antiferromagnetic structures and electronic energy levels at reconstructed NiO(111) surfaces: A DFT + U study. Phys. Rev. B 2015, 91, 235304-235313. [CrossRef]

25. Messiah, A. Quantum Mechanics; North-Holland: Amsterdam, The Netherlands, 1966; Volume II.

26. Sato, H.; Minami, T.; Takata, S.; Yamada, T. Transparent conducting p-type NiO thin films prepared by magnetron sputtering. Thin Solid Films 1993, 236, 27-31. [CrossRef] 
27. Irwin, M.D.; Servaites, J.D.; Buchholz, D.; Leever, B.J.; Liu, J.; Emery, J.D.; Zhang, M.; Song, J.-H.; Durstock, M.F.; Freeman, A.J.; et al. Structural and electrical functionality of NiO interfacial films in bulk heterojunction organic solar cells. Chem. Mater. 2011, 23, 2218-2226. [CrossRef]

28. Garoufalis, C.S.; Poulopoulos, P.; Bouropoulos, N.; Barnasas, A.; Baskoutas, S. Growth and optical properties of $\mathrm{Fe}_{2} \mathrm{O}_{3}$ thin films: A study of quantum confinement effects by experiment and theory. Physica E 2017, 89, 67-71. [CrossRef]

29. Karoutsos, V. Scanning probe microscopy: Instrumentation and applications on thin films and magnetic multilayers. J. Nanosci. Nanotechnol. 2009, 9, 6783-6798. [CrossRef] [PubMed]

30. Peng, H.; Lany, S. Semiconducting transition-metal oxides based on d5 cations: Theory for $\mathrm{MnO}$ and $\mathrm{Fe}_{2} \mathrm{O}_{3}$. Phys. Rev. B 2012, 85, 201202-201207. [CrossRef]

31. Escobar, M.A.; Magana, L.F.; Valenzuela, R. Effect of the grain size distribution on the magnetization curve. J. Appl. Phys. 1985, 57, 2142-2147. [CrossRef]

32. Srolovitz, D.J. Grain growth phenomena in films: A Monte Carlo approach. J. Vac. Sci. Technol. A 1986, 4, $2925-2930$. [CrossRef]

33. Karoutsos, V.; Papasotiriou, P.; Poulopoulos, P.; Kapaklis, V.; Politis, C.; Angelakeris, M.; Kehagias, T.; Flevaris, N.K.; Papaioannou, E.T. Growth modes of nanocrystalline Ni/Pt multilayers with deposition temperature. J. Appl. Phys. 2007, 102, 043525-0435344. [CrossRef]

34. Karoutsos, V.; Toudas, M.; Delimitis, A.; Grammatikopoulos, S.; Poulopoulos, P. Microstructural evolution in nanostructured gold films. Thin Solid Films 2012, 520, 4074-4079. [CrossRef]

35. Zöllner, D. Treating grain growth in thin films in three dimensions: A simulation study. Comput. Mater. Sci. 2016, 125, 51-60. [CrossRef]

36. Atkinson, H.V. Theories of normal grain growth in pure single phase systems. Acta Metall. 1988, 36, 469-491. [CrossRef]

37. Song, X.; Liu, G. Computer simulation of normal grain growth in polycrystalline thin films. J. Mater. Sci. 1999, 34, 2433-2436. [CrossRef]

38. Zhang, J.; Adams, J.B. Modeling and visualization of polycrystalline thin film growth. Comput. Mater. Sci. 2004, 31, 317-328. [CrossRef]

39. Paritosh; Srolovitz, D.J.; Battaile, C.C.; Li, X.; Butler, J.E. Simulation of faceted film growth in two-dimensions: Microstructure, morphology and texture. Acta Mater. 1999, 47, 2269-2281. [CrossRef]

40. Premkumar, P.A.; Delabie, A.; Rodriguez, L.N.J.; Moussa, A.; Adelmann, C. Roughness evolution during the atomic layer deposition of metal oxides. J. Vac. Sci. Technol. A 2013, 31, 061501-061506. [CrossRef]

41. Singh, P.; Chawla, A.K.; Kaur, D.; Chandra, R. Effect of oxygen partial pressure on the structural and optical properties of sputter deposited $\mathrm{ZnO}$ nanocrystalline thin films. Mater. Lett. 2007, 61, 2050-2053. [CrossRef]

42. Seung, C.C.; Koumoto, K.; Hiroaki, Y. Electrical conduction and effective mass of a hole in single-crystal NiO. J. Mater. Sci. 1986, 21, 1947-1950. [CrossRef]

43. Osburn, C.M.; Vest, R.W. Defect structure and electrical properties of NiO-I. High temperature. J. Phys. Chem. Solids 1971, 32, 1331-1342. [CrossRef]

44. Wilson, T.M. A study of the electronic structure of the first-row transition-metal compounds. Int. J. Quantum Chem. 1969, 4, 757-774. [CrossRef]

45. Hanken, H. Kopplung nichtrelativistischer teilchen mit einem quantisierten feld. Nuovo Cim. 1956, 3, 1230-1253. [CrossRef]

46. Mochizuki, S. Radiative optical modes of vibration in NiO thin film. Phys. Status Solidi B 1984, 126, $105-114$. [CrossRef]

47. Lacerda, M.M.; Kargar, F.; Aytan, E.; Samnakay, R.; Debnath, B.; Li, J.X.; Khitun, A.; Lake, R.K.; Shi, J.; Balandin, A.A. Variable-temperature inelastic light scattering spectroscopy of nickel oxide: Disentangling phonons and magnons. Appl. Phys. Lett. 2017, 110, 202406-202410. [CrossRef]

(C) 2018 by the authors. Licensee MDPI, Basel, Switzerland. This article is an open access article distributed under the terms and conditions of the Creative Commons Attribution (CC BY) license (http:/ / creativecommons.org/licenses/by/4.0/). 